\title{
Materials Grindability
}

\author{
Vyacheslav Shumyacher ${ }^{1 *}$, Sergey Kryukov ${ }^{1}$, Olga Kulik $^{1}$ and Xavier Kennedy ${ }^{2}$ \\ ${ }^{1}$ Volzhsky Polytechnic Institute (branch) of Volgograd State Technical University, 404121, 42a, \\ Engelsa street, Volzhsky, Volgograd region, Russian Federation \\ ${ }^{2}$ Carborundum Universal Limited, No.655, T.H.Road, Thiruvottiyur Chennai-600019, India
}

\begin{abstract}
A physical description of material grindability is proposed as a property of deformation and surface destruction of processed surface at the envelope removal. A mechanism for microchip formation is described, which consists in changing mobility of the metal being grinded in "abrasive grain - workpiece" contact, which leads to uneven removal of the energy transmitted by abrasive particle. Criteria for assessing the grindability of metals and methodology for their determination are given. In this article, we described an automated measuring complex (AMC "Grinding"), which was developed, designed and tested by us. This complex is used to solve the aim of the monitoring of grinding process at all stages of abrasive treatment and gives the opportunity to make the adjustments at all stages of the technological process, taking into account both the properties of the abrasive tool and the properties of lubricating and cooling technological means and treatment regimes. The principle of operation of an automated complex is based on statistical analysis of output energy parameters of technological equipment operation. The proposed method for evaluating grindability has been experimentally tested when grinding parts of bearings under production conditions. As a result of processing the obtained data, rational tool characteristics and grinding modes were assigned, providing the required quality indicators.
\end{abstract}

\section{Introduction}

Significant advances in creation of new materials with high values of hardness, modulus of elasticity, melting temperature, wear resistance, low values of linear expansion coefficient, compressibility, thermal conductivity, latent heat served as the basis for manufacture of machine parts operated under extreme conditions. Availability of a complex of physical and mechanical parameters of these materials allows designers to apply them purposefully in design of various technical systems. At the same time, lack of data on processibility of these materials with metal-cutting tools creates difficulties in development of technological process of the parts manufacturing.

With the general trend in machine building to reduce the amount of blade processing to remove significant envelopes, use of high-alloyed and heat-resistant steels and alloys, technical ceramics, the volume of application of abrasive operations increases [1].

\footnotetext{
*Corresponding author:vms22@yandex.ru
} 
Numerous studies have shown that performance and quality of abrasive processing of workpieces from steels and alloys of different composition varies over a wide range [1].

As it follows from work [2], processibility refers to ability of a material to undergo processing, while, along with removal of the envelope, it is necessary to ensure technological requirements for quality and precision of processing. Within the framework of existing ideas [2], grindability of materials is defined as resistance to its destruction while meeting the requirements for accuracy and roughness of processed surface, absence of burns. The author [2] formalized a complex of technological constraints and control parameters, which made it possible to bring the concept of technological processibility to a mathematical description.

At the same time, there is no answer to the question of choosing a quantitative criterion related to the physics of surface strength and workpiece destruction at grinding.

As a criterion characterizing the process of abrasive dispersion of workpiece surface, directly related to surface strength and metal destruction, it was proposed to use specific fracture work, defined as the ratio of work expended on removing the envelope [3] to the volume of material being dispersed.

In order to achieve this goal, the task was to establish a mechanism for micro chip formation at interaction of the workpiece and abrasive grains of the grinding wheel. According to the data of [3], cutting of metals refers to localized high-energy technological methods, accompanied by occurrence of interconnected physical and chemical processes. Earlier [4], a mechanism for chip formation at grinding was proposed. According to [4], the mechanism of metal dispersion by abrasive grains is implemented as follows. Before moving abrasive grains, a shock wave is formed, which has energy up to $70 \mathrm{~kJ} / \mathrm{m}^{3}$, which leads to a loss of metal shear stability.

The transfer rate of disturbances at movement of abrasive grain introduced into the metal will be determined from the dependence [4]:

$$
J=\sqrt{J-2 J V \cos \alpha+V},
$$

where $J$ - is fixed velocity of perturbation of the medium (metal); $V$ - is velocity of movement of abrasive grain; $\alpha$ - is angle between the vector of fixed conduction velocity of disturbances.

Change in the metal mobility leads to uneven removal of the energy transferred by abrasive grain. The amount of energy accumulated in elementary deformable volume of the metal will be determined from the dependence:

$$
\Theta=\frac{d Q}{d t}=\frac{J(X, Y, Z)}{J},
$$

where $d Q$ - is amount of energy accumulated in the volume $d W$.

Increasing the size of abrasive grain leads to an increase in the amount of energy transferred in proportion to the square of the abrasive diameter. Formation of metal chips is a consequence of its transition from a solid to a plastic state due to formation of a shock wave in front of the abrasive grain.

The instability and localization of plastic deformation can be explained by various hypotheses. The main assumptions about the softening of the material are related to the effect of strain rate, temperature, due to energy dissipation, leading to a change in the metal structure [5-10].

For manufacturing machine parts operating under conditions of frictional interaction, complex alloyed compositions and composites with heterogeneous structures are widely used. There are numerous attempts to establish relationship of wear indicators of parts from 
different materials with their physical and mechanical properties and chemical composition. In some cases such a relationship can be traced by selecting the conditions of laboratory tests.

Unfortunately, at present there is no scientifically based method for assessing grindability of steels, alloys, composites, which allows to establish the influence on it of the physical and mechanical properties of the materials being processed. As a consequence, the use of new promising materials is complicated because of a need for long-term experimental studies in development of technological process of manufacturing parts, in particular, assignment of grinding modes and tools characteristics.

Works of V.D. Kuznetsov [11] established the proportionality between the volume of metal dispersed by abrasive sandpaper and the work of friction. As a criterion, the ratio of the work expended on removing the mass of metal at abrasive dispersion to this mass was used.

However, according to [11], this approach was considered unpromising, since its effect extended to all cases of surface dispersion without taking into account the nature of the phenomena causing the processes of wear, grinding, cutting, chipping [3]. Research conducted by B.I.Kostetskiy and his scientific school [3] substantiated the energy connection of the work of friction forces and surface destruction of materials and proved the possibility of using specific work of wear as a complex physical criterion.

Application of this criterion allows the selection of materials for friction pairs, lubricants, loading modes [3]

Energy methods for estimating wear rates have been used in tribology for many decades and were first formulated in the works of Fleischer [11]. According to this theory, separation of a particle of material wear is a consequence of accumulation of material, in a certain volume, in the surface layer of a certain critical reserve of internal energy. V.V. Fedorov [11] proposed to estimate the rate of abrasive wear at friction of the sample on the abrasive wheel using the formula (3):

$$
\dot{V}=\frac{n S}{t}=\frac{\dot{U}_{e}}{U_{*}-U_{T f}},
$$

where $n, S, t-$ is, respectively, the number of revolutions of abrasive wheel, contact surface area of the wheel and workpiece, operating time; $U_{*}-$ is critical density of material energy; $U_{T f}$-is density of the thermal component of latent energy; $\dot{U}_{e}$ - is the rate of change of latent energy in the surface layer of material interacting with the abrasive wheel.

$\dot{U}_{e}$ - can be calculated by dependencies:

$$
\dot{U}_{e}=\delta_{e} W,
$$

where $\delta_{e}$ - is relative magnitude of the latent energy; $W$-is power density of friction.

According to [11], the concept of metal processability by cutting implies a set of interrelated material properties responsible for removing the envelope, which is reduced to the property of the metal to undergo deformations and destruction when the tool affects the workpiece.

This property is opposite to the property of material to resist the explosive nature of fracture at cutting (process time $10^{-4}-10^{-7} c$, volumetric energy release $2 \ldots 70 \mathrm{j} / \mathrm{mm}^{3}$; pressure up to 10 - $70 \mathrm{GPa}[12]$.

When cutting metals at roughing and semi-finishing, processability characteristic is the value of permissible cutting speed $V_{T}$, corresponding to the standard value of the tool life period $T$ [11]. A material that allows a higher cutting speed has a better processability [12]. 
Experimental dependencies were obtained that connect the allowable cutting speed with metal hardness $\left(H B, H R C, H_{100}\right)$, yield strength $\left(G_{T}\right)$, temporary resistance $\left(G_{B}\right)$, actual tensile strength $\left(S_{B}\right)$, thermal conductivity $(\lambda)$, chemical composition [13]. Unfortunately, none of these characteristics of metal processability by cutting does not fully meet the requirements of practice.

The energy supplied by abrasive grains of the grinding wheel to the surface of the workpiece is divided into "useful", spent on solving technological problems and converts the properties of metal. Another part of energy is dissipated or contributes to the wear of the instrument (microcleaving (microchipping) of abrasive grains, falling out of the ligament), as well as transformation of the metal structure, which causes a change in the physical and mechanical properties: hardness, microhardness, microplasticity. Grindability of metal can be considered as the ability, under the conditions of physicochemical and physicomechanical effects accompanying abrasive dispersion, to provide the required parameters of the processed surface of the workpiece. It can be stated that grindability of metal is an integral energy characteristic.

As a result of interaction of the grinding wheel with the workpiece surface, microchip is formed. The ratio of volume of the envelope removal of the workpiece to the energy value is an important indicator - specific work of dispersion. It can be assumed that a decrease in the specific work of dispersion indicates an improvement in processability of the workpiece of metal.

In accordance with [13], at grinding, external work is spent on dispersing the surface layer, elastic compressive or shear deformations, as well as plastic deformations of the same types, the total heat flow released in the "tool-workpiece" contact at grinding is determined from dependence [13] :

$$
q_{k}+q_{\partial}+q_{c}=\frac{F_{z}(V) \cdot V}{S J},
$$

where $S$-is actual area of discrete contact; $V=V_{k}=V_{\partial}$ - is cutting speed, i.e. relative speed of the wheel and workpiece; $J$ - is mechanical equivalent of heat (in the SI system $J=1$ ); $F_{z}$-is tangential component of cutting force; $q_{k}+q_{\partial}+q_{c}$ - is heat flow carried away by a wheel, detail, chips.

The dispersion products formed during grinding process carry away the thermal power from the contact $S[13]$ :

$$
q_{c}=\frac{C_{\partial} m_{\partial} T_{\partial}\left(O, Z_{0}\right)}{S}
$$

where $m_{\partial}-$ is the mass of remote envelope; $C_{\partial}-$ is heat capacity of the workpiece; $C_{\partial}=\frac{\lambda_{\partial}}{d_{\partial} \rho_{\partial}}$, где $\lambda_{\partial}$ - is thermal conductivity of the workpiece, $d_{\partial}-$ is thermal diffusivity; $\rho_{\partial}$ - is density of the workpiece material; $Z_{0}$ - is the point on the "wheel - workpiece" contact, where the maximum temperature is reached.

According to [13], at grinding, the heat flow $\left(q_{k}\right)$ carried away by the grinding wheel does not depend on the contact temperature, since it is determined only by geometrical parameters of the contact zone of the tool and the workpiece and their thermophysical characteristics.

The value of $q_{k}$ increases with increasing thermal conductivity of the grinding wheel and decreases with increasing thermal conductivity of the workpiece.

The heat flow at the "grinding wheel -workpiece" contact is distributed in accordance with the dependencies [13]: 


$$
Q_{k}(0,0)=\left(1-K_{1}\right) q ; \quad q \partial(0,0)=K_{1} K_{2} q ; q_{c}(0,0)=K_{1}\left(1-K_{2}\right) q
$$

In turn, $K_{1}$ and $K_{2}$ is determined from the equations [27]:

$$
\begin{aligned}
& K_{1}=\frac{f_{k}(O, Z)}{f_{k}(O, Z)+f_{\partial}(O, Z)}, \\
& K_{2}=\frac{1}{1+\frac{\lambda_{\partial} V_{\partial} l b}{d_{\partial} S} f_{\partial}(O, Z)},
\end{aligned}
$$

where $b$ - is the width of the grinding wheel; $l$ - is the depth of cut; $f_{k}(O, Z), f_{\partial}(O, Z)$ - are coordinate functions of temperature distribution on the surface of the wheel and the workpiece; $d_{\partial}$ - thermal diffusivity of the workpiece material.

In accordance with the data of $[14,15]$, a change in the physical and mechanical properties of the workpiece material is a reflection of its internal response to the impact of external energy and ensuring production of defect-free parts is the correct transformation and quantitative distribution of the input energy and the rational table mode.

Guided by the above considerations, grindability of a workpiece from a specific material can be estimated by the value of specific work of dispersion at grinding, which is determined by the integral function of the thermal energies emitted by the tool $\left(q_{k}\right)$, surface of the workpiece $\left(q_{\partial}\right)$ and dispersion products $\left(q_{c}\right)$.

The heat flow into the surface layer of the workpiece is reduced by the value of $q_{c}$; therefore, a decrease in specific energy of dispersion indicates improvement in grindability of the material. Quantitative evaluation of $q_{k}, q_{\partial}, q_{c}$ can be determined using dependencies (7), (8), (9).

\section{Practical testing}

For practical assessment of grindability of metals, an automated measuring complex (AMC "Grinding") has been developed, manufactured and tested, which allows monitoring the process with determining specific energy of dispersion over a processing cycle with a time step of $0.01 \mathrm{~s}$ and tool wear.

The proposed method for evaluating grindability has been experimentally tested when grinding parts of bearings under production conditions. As a result of processing the obtained data, rational tool characteristics and grinding modes were assigned, providing the required quality indicators.

\section{References}

1. S.N. Korchak, Bulletin of mechanical engineering, 11, 52 (1968)

2. A.A. Dyakonov, Bulletin of the South Ural State University. Series "Mechanical Engineering Industry", 15:1, 54 (2015)

3. V.M. Yaroslavtsev Science and Education, 1, 1 (2011)

4. V.M. Shumyacher, A.V. Kadilnikov, Engineering Technology, 4, 8 (2007)

5. K.Nadolny, W.Kaplonek, G. Krolczyk, N. Ungureanu, Journal of Engineering Manufacture 232:6, 965 (2018)

6. W. Lothar, E. Staskewitsch, A. Burblies, Mechanics of Materials, 17:2-3, 203 (1994) 
7. B. Gardiner, A. Tordesillas, International Journal of Solids and Structures, 41:21, 5885 (2004)

8. C. Fressengeas, A. Molinari, Journal of the Mechanics and Physics of Solids, 40:8, $1735(1992)$

9. C. Fressengeas, A. Molinari, Journal of the Mechanics and Physics of Solids, 35:2, 18 (1987)

10. M.A. Meyers, G. Subhash, B.K. Kad, L. Prasad, Mechanics of Materials 17:2, 175 (1994)

11. V.M. Yaroslavtsev, Vestnik MGTU. Ser. "Machine building", 4, 32 (2000)

12. V.M. Yaroslavtsev, Energy characteristic of machinability (Science and education, Electronic scientific and technical edition, 2012)

13. D.G. Evseev, A.N. Salnikov, Grinding models. Theory and experiment. Monograph. (Moscow, Izvestia Publishing House, 2018)

14. V.M. Yaroslavtsev, Technological process - energy converter (Science and education, Electronic scientific and technical edition, 2012)

15. V. M. Shumyacher, A. V. Slavin, O. G. Kulik, LNME, 153, 1443 (2019) 